Erwăgung, daß sich in der letzten Zeit mehr und mehr Beziehungen zwischen der. chemischen Struktur und der kristallographischen Form herausgestellt haben, und daB wir nach der Gesamtheit unserer chemischen Kenntnisse annehmen müssen, daB die Kräfte, welche chemische Systeme (Verbindungen) im Gleichgewicht halten, von der Richtung im Raume abhängen, so müssen wir schließen, daß die kleinsten Massen irgend eines Stoffes, welche die individuellen Eigenschaften derselben noch nicht verloren haben, vektorial gebaut sind. Betrachten wir weiter die Tatsache, daß die weichen und selbstverständlich auch die flüssigen Kristalle, wie z. B. das oleïnsaure Kalium sogar bei großen Dimensionen die Eigenschaft besitzen, sich bei unmittelbarer Berührung zu einem homogen kristallinischen Ganzen zu vereinigen, so ist ersichtlich, daß die elementaren kristallinischen Massen eine große Beweglichkeit besitzen können, ohne ihre vektoriale Verkettung zu verlieren. Auf Grund dieser Darlegung folgt also in theoretischer Beziehung:

5. Die kristallinische Struktur ist durch die innerliche Symmetrie der elementaren kristallinischen Massen des Stoffes bedingt. Diese Symmetrie verschwindet nicht beim Uebergang in den flüssig-gasförmigen Zustand (bei dem aber eine Umlagerung der Symmetrie stattfinden kann); nur zwingt die Steigerung der Beweglichkeit (oder der Weglängen) die kristallinischen Massen von Richtungen, die parallel $\mathrm{zu}$ ihren Symmetrieelementen verlaufen, abzuweichen. Die Zähigkeit von Lösungen, welche große Mengen festen Stoffes enthalten und die Zähigkeit der Flüssigkeiten in der Nähe ihres Erstarrungspunktes wird bedingt durch das Bestreben der elementaren Massen, sich parallel zueinander zu bewegen, ein Bestreben, welches bei den betreffenden Druck- und Temperaturbedingungen nicht erfüllt werden kann. - Aus diesen Gründen müssen identische Bedingungen für den Uebergang gasförmig-flüssig sowie für den Uebergang flüssig-fest existieren.

Ganz im allgemeinen folgt noch aus diesen Untersuchungen, daß kolloide und kristalloide keineswegs $z$ wei besondere Welten sind, sondern, daB zwischen ihnen selbst sowohl, als auch zwischen ihnen und den gasförmigen und flüssigen Zuständen der Materie, enge Beziehungen bestehen.

September 1905 - Mai 1907.

St. Petersburg. Chem. Labor, d. k. Berginstitutes Kaiserin Katharina II.

\title{
Ueber den Einfluß von Salzen auf den Färbungsvorgang.
}

Von L. Pelet und L Grand.

Durch die Arbeiten von Biltz1) wurde die Analogie zwischen dem Verhalten des Benzopurpurin, eines direkten Farbstoffes, und den Eigenschaften kolloider Lösungen besonders klargelegt, obschon man seit den Untersuchungen von Krafft bereits wuBte, daß die direkten Farbstoffe sich im Zustand kolloider Lösung befanden. In der Tat liefern die sauren und basischen Farbstoffe Lösungen, die wesentlich von denen der direkten Farbstoffe verschieden sind; sie sind optisch leer und lassen sich nicht dialysieren.

Die verschiedensten Autoren, die sich mit Kolloiden beschäftigten (Perrin, Henry, Lottermoser etc.) haben die Meinung geäußert, daß die Kolloide eine ganz hervorragende Rolle bei der Fixierung von Farbstoffen durch die Faser

I Biltz: Beri. Ber. 1905, S. 2963 etc. spielen müBten. Auch Freundlich und Losev gelangen mittels einer Reihe von Deduktionen zu dem Schlusse, dab der Färbungsvorgang als ein Fallungsvorgang von Kolloiden auf die Faser oder auf die Substanz, welche făhig ist, den Farbstoff zu adsorbieren, aufzufassen ist.

Wir haben nun versucht, diese Theorie in Bezug auf die Färbung durch Kristallponceau (einen sauren Farbstoff) und durch Methylenblau (einen basischen Farbstoff) zu verifizieren.

Man weiB, daß die Fällung kolloider Lö. sungen stark aktiviert (oder beschleunigt) wird durch den Zusatz polyvalenter Ionen mit entgegengesetzter Ladung. In diesem Sinne haben wir nun untersucht, wie die Färbung mittels Kristallponceau und Methylenblau durch Salze beeinfluBt wird. Die Resultate sind in den folgenden Tabellen enthalten. 
Tabelle 1.

$2 \mathrm{~g}$ Wolle in $200 \mathrm{ccm}$ einer $0,5 \%$ Methylenblaulösung.

\begin{tabular}{l|c}
\hline Zusaiz zum Bad & $\begin{array}{c}\text { Adsorbierte Menge in } \\
\mathrm{mg}=\mathrm{x}\end{array}$ \\
\hline $0,3 \mathrm{~g} \mathrm{Na} \mathrm{SO}_{4}$ & 77,7 \\
$0,3 \mathrm{~g} \mathrm{Na} \mathrm{HPO}_{4}$ & 97,5 \\
$0,2 \mathrm{~g} \mathrm{BaCl}$ & 68,7 \\
2 Tropfen Pt Cl $\mathrm{H}_{3}$ & $79,2(\mathrm{l})$ \\
ohne Zusatz & 74,5
\end{tabular}

Der Wert 79,2 (für Platinchlorür) ist das Mittel aus mehreren Gehaltsbestimmungen mittels Kristallponceau. Wir betrachten diesen Wert als falsch, da die Titration stark von dem Platinchlorür oder der Chlorsäure oder durch beide gleichzeitig beeinfluBt werden mub. Als Beweis hierfür kann die Farbe der Wolle, welche nach dem Entfernen aus dem Bad ein viel helleres Aussehen ats die andern Proben hatte, argesehen werden.

Die Färbungen der Wolle nach Auswaschen und Trocknen verhielten sich folgendermaßen : Die Wolle im Bad mit $\mathrm{Na}_{2} \mathrm{SO}_{4}$ hatte eine dunklere Färbung als die Probe ohne Zusatz; die Wolle mit $\mathrm{Na}_{2} \mathrm{HPO}_{4}$ war noch dunkler als die vorhergehende und zeigte braunrote Reflexe (Bronzefarbe), wie sie bei den festen Kristallen des Methylenblaus bekannt sind. Im Gegensatz hierzu hatte die Wolle in Gegenwart von $\mathrm{BaCl}_{\mathrm{a}}$ eine viel hellere Färbung angenommen als die ohne Zusatz, und die in Gegenwart von $\mathrm{PtCl}_{4}$ gefärbte Wolle war endlich noch viel heller.

Gehaltsbestimmungen, welche wir mittels der Methode von Knecht ausführten, gaben Resultate von analoger Beschaffenheit und gleicher Crößenordnung; einige Resultate mit Kristallponcean sind in Tabelle 2 nitgeteilt.

Tabelle 2.

$2 \mathrm{~g}$ Wolle in $200 \mathrm{ccm}$ einer $0,5 \%$ Kristalponceaúösung.

\begin{tabular}{l|c}
\hline Zusatz zun Bat & $\begin{array}{c}\text { Adsorbierte Menge in } \\
\text { mig =x }\end{array}$ \\
\hline $0,3 \mathrm{~g} \mathrm{Na}_{2}, \mathrm{SO}_{4}$ & 11,4 \\
$0,3 \mathrm{~g} \mathrm{Na} \mathrm{HPO}_{4}$ & 0 \\
2 Tropien $\mathrm{PtCl}_{4}$ & 20 \\
che Zusatz & 13,1
\end{tabular}

In diesem Falle zeigen die Beobachtungen also das Gegenteil zum vorigen: Die Wolle ist deutlich weniger in Gegenwart von $\mathrm{Na}_{2} \mathrm{SO}_{4}$ gefärbt und nur schwach rosa bei Zusatz yon $\mathrm{Na}_{2} \mathrm{HPO}_{4}$, während sie viel dunkler durch Znsatz von $\mathrm{PtCl}_{4}$ gefärbt wurde. Ein Versuch mit $\mathrm{Ba} \mathrm{Cl}_{2}$ konnte darum nicht gemacht werden, weil dieses Salz das Kristallponceau aus seiner Lösung ausfällt.

Tabelle 3.

$1 \mathrm{~g}$ kalzinierte Kieselsäure in $200 \mathrm{ccm} 1 \%$ Methylenblaulösung.

\begin{tabular}{c|c}
\hline Zusatz zum Bad & $\begin{array}{c}\text { Adsorbiette Menge in } \\
\text { mg }=\mathbf{x}\end{array}$ \\
\hline $0,2 \mathrm{~g} \mathrm{Na}_{2} \mathrm{SO}_{4}$ & 69,6 \\
$0,2 \mathrm{~g} \mathrm{Na} \mathrm{HPO}_{4}$ & 79,7 \\
$0,2 \mathrm{~g} \mathrm{Ba} \mathrm{Cl}$ & 64 \\
ohne Zusatz & 67
\end{tabular}

Tabelle 4

$1 \mathrm{~g}$ Kohle in $200 \mathrm{ccm}$ einer $1 \%$ Kristallponceaulösung.

\begin{tabular}{l|c}
\hline Zusatz zum Bad & $\begin{array}{c}\text { Adsorbierte Menge in } \\
\text { mg }=\mathrm{x}\end{array}$ \\
\hline $0,2 \mathrm{~g} \mathrm{Na}_{2} \mathrm{SO}_{1}$ & 67,5 \\
$0,2 \mathrm{~g} \mathrm{Na} \mathrm{HPO}_{4}$ & 57,5 \\
ohne Zusatz & 70,1
\end{tabular}

Diese Resultate beweisen in der Tat, daß der Färbevorgang eine Fällung der Farbkolloide auf die Faser oder den adsorbierenden Körper ist.

Die Fällung des Methylenblaus, betrachtet als ein positives Kolloid, wird befördert durch Zusatz bivalenter lonen $\left(\mathrm{SO}_{4}\right)$ und besonders durch trivalente negative lonen $\left(\mathrm{PO}_{4}\right)$. In Gegensatz hierzn setzen die lonen mit demselhen Vorzeichen (das bivalente $\mathrm{Ba}$ und das tetravalente $P_{1}$ ) die Adsorption herab und zwar umsonehr, je höher die Valenz ist.

Auf der anderen Seite wird die Adsorption des Kristallponceaus, eines negativen Kolloids, herabgesetzt durch Ionen mit gleichen Vorzeichen, dem bivalenten $\mathrm{SO}_{4}$ und vor allen - Dingen dem trivalenten $\mathrm{PO}_{4}$; umgekehrt wird dieselbe erhöht durch lonen nit entgegengesetzten Vorzeichen.

Laboratoire de Chimie Industrielle de l'Universite de Lausanne. 